\title{
Proteomic study to identify factors in follicular fluid and/or serum involved in in vitro cumulus expansion of porcine oocytes
}

\author{
J. Bijttebier', K. Tilleman², D. Deforce², M. Dhaenens' ${ }^{2}$, A. Van Soom ${ }^{1}$ \\ and D. Maes' \\ 'Department of Reproduction, Obstetrics and Herd Health, Faculty of Veterinary Medicine, \\ Chent University, Merelbeke, Belgium; 'Laboratory for Pharmaceutical Biotechnology, Faculty of \\ Pharmaceutical Sciences, Chent University, Chent, Belgium
}

Follicular fluid, as a transudate of serum, constitutes the micro environment of the maturing oocyte. In a previous study, we have shown that follicular fluid (pFF) is superior to autologous serum in promoting cumulus expansion during in vitro maturation (IVM) of porcine oocytes (Bijttebier et al. 2008). After ultrafiltration of both fluids, the fraction containing molecules $>30 \mathrm{kDa}$ includes the factor(s) responsible for the observed differences in cumulus expansion (f Bijttebier, unpublished observations). This suggests the factor is likely to be a protein. The present study aimed to identify those proteins responsible for the observed differences in cumulus expansion after IVM in $10 \%$ serum ( $>30 \mathrm{kDa}$ ) versus $10 \% \mathrm{pFF}$ ( $>30 \mathrm{kDa}$ ) obtained from sows in the preovulatory stage of the estrous cycle.

Shotgun proteomics analysis of the pFF and serum fractions $>30 k D a$ from 3 sows was performed by application of the 'isobaric Tag for Relative and Absolute Quantitation'(iTRAQ) technology (Applied Biosystems) followed by 2D-LC ESI-Q-TOF MSMS. Of each sample, 100 $\mu \mathrm{g}$ protein material was loaded and runs were performed in duplicate. The processed data, obtained from Mascot Daemon, was searched against the pig EST database for protein identification (http:// pigest.ku.dk). Protein ratios resulting from duplicate runs were averaged, log-transformed and analyzed by Student's t-test. In addition, 600 prepubertal gilt oocytes were matured in vitro for $26 \mathrm{~h}$ in NCSU23 supplemented with $10 \% \mathrm{pFF}$ ( $>30 \mathrm{kDa})$ or $10 \%$ serum $(>30 \mathrm{kDa})$ of each of the 3 sows. After IVM, the expanded cumulus matrices were collected and subjected to proteomic analysis. Proteins in the matrix extracts were separated using 2D-PACE. Two spots that were absent in matrices matured in pFF were excised and submitted to mass spectrometric analysis using ESI-Q-TOF MSMS. The processed data, obtained from Mascot Daemon, was searched against the pig EST database for protein identification.

First of all, serum and pFF were not depleted for high abundant proteins like albumin, because the depleted sample did not show the same biological effect on the IVM of porcine oocytes. Therefore an exclusion list was used based on the first run to exclude abundant peptides derived from albumin. Proteomic analysis of serum and pFF revealed 63 unique proteins present in both fluids of which 13 showed significantly $(P<0.05)$ different expression levels (10 proteins levels on $P<0.01$ ). Seven of these proteins were more abundant in serum whereas 6 of them were more abundant in the pFF fractions. Alpha2-macroglobulin (A2M) and ch4 and secrete domains of swine IgM, which were both down regulated in $\mathrm{pFF}$, were also identified as the protein spots that were absent in the cumulus matrices after IVM in $10 \%$ pFF (>30 kDa) compared to IVM in $10 \%$ serum (>30 kDa).

In conclusion, 2 proteins that are upregulated in autologous serum were also solely retrieved in the cumulus matrices of oocytes matured in $10 \%$ serum ( $>30 \mathrm{kDa}$ ). One of them, A2M, 
might be involved in the observed differences in cumulus expansion. Alpha2- macroglobulin, as a major proteinase inhibitor, might degrade ADAMTS-1, which has been shown to be essential for gonadotropin-regulated cumulus expansion of porcine cumulus-oocyte-complexes (Shimada et al. 2004). However, further experiments should be performed to elucidate the mechanisms responsible for the possible inhibiting effects of $A 2 M$ on cumulus expansion.

Table 1: Differentially expressed proteins $(p<0.05)$ in autologous serum and pff of three sows in the preovulatory phase of the estrous cycle. Protein ratio values in italics correspond to proteins that are more abundant in pff compared to serum

\begin{tabular}{lcc}
\hline Protein & Protein ratio pFF/serum & P-value protein ID \\
\hline$\alpha 2$-macroglobulin & 0.274 & 0.000 \\
apolipoprotein CIII & 0.375 & 0.000 \\
angiopoetin-like protein 2 & 11.783 & 0.011 \\
ch4 and secrete domains of swine IgM & 0.201 & 0.000 \\
clusterin & 1.334 & 0.050 \\
complement component C4 & 0.893 & 0.000 \\
fibrinogen $\beta$ chain & 1.607 & 0.014 \\
IgG $\alpha$ chain C region & 0.804 & 0.008 \\
IgG E $\varepsilon$ chain & 0.916 & 0.002 \\
inter- $\alpha$-globulin inhibitor HI & 1.175 & 0.001 \\
antithrombin III & 1.465 & 0.000 \\
angiotensinogen & 1.117 & 0.08 \\
IgG VDJ chain & 0.846 & 0.001 \\
\hline
\end{tabular}

\section{References}

Bijttebier J, Van Soom A, Meyer E, Mateusen B \& Maes D 2008 Preovulatory follicular fluid during in vitro maturation decreases polyspermic fertilization of cumulus-intact porcine oocytes. Theriogenology 70 715-724.
Shimada M., Nishibori M, Yamashita Y, Ito J, Mori T \& Richards IS 2004 Down-regulated expression of ADAMTS-1 by progesterone receptor antagonist is associated with impaired expansion of porcine cumulus-oocyte complexes. Endocrinology 145 4603-4614. 